\title{
TeV SUSY dark matter confronted with the current direct and indirect detection data
}

\author{
Murat Abdughani ${ }^{1,2, a} \mathbb{D}$, Jie Ren ${ }^{1,2}$, Jun Zhao ${ }^{1,2,3}$ \\ ${ }^{1}$ CAS Key Laboratory of Theoretical Physics, Institute of Theoretical Physics, Chinese Academy of Sciences, Beijing 100190, China \\ ${ }^{2}$ School of Physical Sciences, University of Chinese Academy of Sciences, Beijing 100049, China \\ ${ }^{3}$ Institute of Theoretical Physics, College of Applied Science, Beijing University of Technology, Beijing 100124, China
}

Received: 28 May 2018 / Accepted: 4 February 2019 / Published online: 19 February 2019

(C) The Author(s) 2019

\begin{abstract}
In the minimal supersymmetric standard model (MSSM) the lightest superparticle (LSP) can be a TeV neutralino (mainly dominated by higgsino or wino) which serves as a dark matter candidate with correct thermal relic density. In this work we confront the $1-3 \mathrm{TeV}$ neutralino dark matter with the latest direct and indirect detections from PandaX and AMS-02/DAMPE. Considering various scenarios with decoupled sfermions, with $A$-mediated annihilation, with squark or stop coannihilation, we find that the parameter space is stringently constrained by the direct detection limits. In the allowed parameter space, the $\mathrm{TeV}$ neutralino dark matter annihilation contribution to the anti-proton flux is found to agree with the AMS-02 data while its contribution to eletron/positron flux is too small to cause any visible excess.
\end{abstract}

\section{Introduction}

Identifying the nature of the cosmic dark matter is a primary topic in today's particle physics and cosmology. In the popular minimal supersymmetric standard model (MSSM), the lightest neutralino is a natural candidate for the cosmic cold dark matter. In general, a neutralino dark matter with a mass around $100 \mathrm{GeV}$ (a typical WIMP) can be effectively explored at direct detection experiments and the LHC. However, a neutralino dark matter above $\mathrm{TeV}$ scale or below $\mathrm{GeV}$ scale is hard to detect at the LHC. On the other hand, the current direct detections [1] have relatively low sensitivities to such a super-heavy or ultra-light neutralino dark matter and hence the limits on their interactions with the nucleon are rather weak. For the TeV scale dark matter, another motivation comes from the recent DAMPE observation [2] of a plausible electron/positron excess at TeV energy which may

\footnotetext{
a e-mail: mulati@itp.ac.cn
}

indicate a heavy dark matter at $\mathrm{TeV}$ scale. Theoretically, in the MSSM an ultra-light GeV scale neutralino dark matter can only be achieved in some unnatural limits (say the alignment limit without decoupling [3,4]), but a TeV scale neutralino dark matter can be naturally obtained with correct thermal relic density. So a TeV scale neutralino dark matter is an interesting scenario to study.

Such a TeV neutralino dark matter has been discussed in the literature [5-16]. In this work we intend to give a more complete study by considering various scenarios with decoupled sfermions, with $A$-mediated annihilation, with squark or stop coannihilation. Under the requirement of giving correct thermal relic density, we will show its components. Then we will demonstrate the constraints of the latest direct detections on its parameter space. In the allowed parameter space we will show the contributions of its annihilation to the antiproton and eletron/positron cosmic-ray fluxes, which will be compared with the AMS-02 and DAMPE data.

The structure of this paper is organized as follows. In Sect. 2 we show the scenarios and the components of the $\mathrm{TeV}$ neutralino dark matter under the requirement of giving correct thermal relic density. In Sect. 3 we confront the TeV neutralino dark matter in various scenarios with the latest direct detection limits from PandaX and indirect constraints from the anti-proton and eletron/positron cosmic-ray fluxes from AMS-02 and DAMPE data. Finally, we draw our conclusions in Sect. 4.

\section{$2 \mathrm{TeV}$ neutralino dark matter with correct relic density}

In the MSSM the two neutral higgsinos $\left(\tilde{H}_{u}^{0}\right.$ and $\left.\tilde{H}_{d}^{0}\right)$ and the two neutral guaginos $\left(\tilde{B}\right.$ and $\left.\tilde{W}^{0}\right)$ are mixed to form four mass eigenstates called neutralinos. In the gauge-eigenstate basis $\left(\tilde{B}, \tilde{W}^{0}, \tilde{H}_{d}, \tilde{H}_{u}\right)$, the neutralinos are defined as $\tilde{\chi}_{i}^{0}=$ $Z_{N}^{i j}\left(\tilde{B}, \tilde{W}^{0}, \tilde{H}_{d}, \tilde{H}_{u}\right)$ while the charginos are defined as 
$\tilde{\chi}_{i}^{ \pm}=Z_{ \pm}^{i j}\left(\tilde{W}^{ \pm}, \tilde{H}^{ \pm}\right)$, where $\tilde{B}, \tilde{W}, \tilde{H}_{d}, \tilde{H}_{u}$ are respectively the bino, wino, and higgsino fields, and $Z_{N}^{i j}$ and $Z_{ \pm}^{i j}$ are neutralino and chargino mixing matrices.

In our analysis we take the bino mass $M_{1}$, wino mass $M_{2}$, and higgsino mass $\mu$ in the range of $1-10 \mathrm{TeV}$ when we scan over the parameter space. We require the neutralino dark matter (the lightest neutralino) in the range of 1-3 TeV. In our scan we use MicrOEMGAs [17] to calculate the thermal relic density $\Omega_{\tilde{\chi}} h^{2}$ and the cross sections and require the neutralino dark matter to provide the relic density in the $2 \sigma$ range of the measured value [18]. We choose vacuum expectation values $5<\tan \beta<60$ and trilinear terms $A_{i}=0$ if not mentioned below. We consider the following scenarios:

1. Decoupled case Motivated from the split supersymmetry [19], in this case we set sfermion mass parameters and the CP-odd Higgs mass as heavy as $10 \mathrm{TeV}$ to decouple them from gauginos. In this case the main processes which affect the dark matter relic density and annihilation cross section involve the interactions between neutralinos, charginos and the $W / Z$ or Higgs boson. The interactions of gauge boson with neutralino and chargino are given by (we used the conventions in [20] for particles, couplings, and their diagonalization matrices)

$$
\begin{aligned}
& \frac{e}{s_{W}} \bar{\chi}_{j} \gamma^{\mu}\left[\left(Z_{N}^{2 i} Z_{+}^{1 j \star}-\frac{1}{\sqrt{2}} Z_{N}^{4 i} Z_{+}^{2 j \star}\right) P_{L}\right. \\
& \left.+\left(Z_{N}^{2 i \star} Z_{-}^{1 j}+\frac{1}{\sqrt{2}} Z_{N}^{3 i \star} Z_{-}^{2 j}\right) P_{R}\right] \chi_{i}^{0} W_{\mu}^{+}-\frac{e}{2 s_{W} c_{W}} \bar{\chi}_{i} \gamma^{\mu} \\
& \times\left(Z_{+}^{1 i \star} Z_{+}^{1 j} P_{L}+Z_{-}^{1 i} Z_{-}^{1 j \star} P_{R}+\left(c_{W}^{2}-s_{W}^{2}\right) \delta^{i j}\right) \chi_{j} Z_{\mu} \\
& +\frac{e}{4 s_{W} c_{W}} \bar{\chi}_{i}^{0} \gamma^{\mu}\left(\left(Z_{N}^{4 i \star} Z_{N}^{4 j}-Z_{N}^{3 i \star} Z_{N}^{3 j}\right) P_{L}\right. \\
& \left.-\left(Z_{N}^{4 i} Z_{N}^{4 j \star}-Z_{N}^{3 i} Z_{N}^{3 j \star}\right) P_{R}\right) \chi_{j}^{0} Z_{\mu}
\end{aligned}
$$

where $\chi_{i}^{0}, i=1 \ldots 4$ is neutralinos, $i=1$ neutralino is LSP, $\chi_{i}, i=1,2$ is charginos. $Z_{N}^{i j}, i, j=1 \ldots 4$ is $j$ th, in mass ascending order, neutralino's $i$ th component, $i=1$ is bino, $i=2$ is wino, $i=3,4$ is two higgsino components. $Z_{ \pm}^{i j}, i, j=1,2$ is $j$ th, in mass ascending order, chargino's $i$ th component, $i=1$ is wino, $i=2$ is higgsino component. $P_{L}=\left(1-\gamma^{5}\right) / 2, P_{R}=(1+$ $\left.\gamma^{5}\right) / 2 . s_{W}=\sin \theta_{W}, c_{W}=\cos \theta_{W}, \theta_{W}$ is weak mixing angle. The interactions of Higgs boson with neutralino and chargino are given by

$$
\begin{aligned}
& \frac{e}{2 s_{W} c_{W}} \bar{\chi}_{i}^{0}\left[\left(Z_{R}^{11} Z_{N}^{3 j}-Z_{R}^{21} Z_{N}^{4 j}\right)\left(Z_{N}^{1 i} s_{W}-Z_{N}^{2 i} c_{W}\right) P_{L}\right. \\
& \left.+\left(Z_{R}^{11} Z_{N}^{3 i \star}-Z_{R}^{21} Z_{N}^{4 i \star}\right)\left(Z_{N}^{1 j \star} s_{W}-Z_{N}^{2 j \star} c_{W}\right) P_{R}\right] \chi_{j}^{0} h \\
& -\frac{e}{\sqrt{2} s_{W}} \bar{\chi}_{i}\left[\left(Z_{R}^{11} Z_{-}^{2 i} Z_{+}^{1 j}+Z_{R}^{21} Z_{-}^{1 i} Z_{+}^{2 j}\right) P_{L}\right. \\
& \left.+\left(Z_{R}^{11} Z_{-}^{2 j \star} Z_{+}^{1 i \star}+Z_{R}^{21} Z_{-}^{1 j \star} Z_{+}^{2 i \star}\right) P_{R}\right] \chi_{j} h
\end{aligned}
$$

where $Z_{R}^{11}=Z_{R}^{22}=\cos \alpha, Z_{R}^{12}=-Z_{R}^{21}=-\sin \alpha$, $\tan 2 \alpha=\tan 2 \beta \times\left(M_{A}^{2}+M_{Z}^{2}\right) /\left(M_{A}^{2}-M_{Z}^{2}\right)$.

We know from the above equations that the pure bino LSP $\left(\chi_{1}^{0}\right)$ does not interact with gauge boson or Higgs boson. So the bino LSP annihilation can only proceed through coannihilation or mixing with higgsino or wino. Thus the bino dark matter annihilation cross section is smaller than higgsino and wino dark matter. For pure bino LSP, it is highly mass degenerate with lighter chargino, coannihilation of these two particle contribute sifnificantly to the DM relic density. Further more, Sommerfeld enhancement must be taken to the account which increase the annihilation of DM, as the result, wino mass satisfies corrent relic density becomes larger. If LSP is pure higgsino, then $m_{\chi_{1}^{0}} \simeq m_{\chi_{2}^{0}} \simeq m_{\chi_{1}}$, higgsino efficiently annihilate through $s$-channel $Z$ diagram, besides coannihilation within the two neutralinos and one chargino contributes to the relic density.

2. A-mediated case In this case the LSP dark matter annihilates through the $s$-channel resonance of the CP-odd Higgs boson $A$, usually called " $A$-funnel" [21]. Here we decouple all sfermions (fix them to $10 \mathrm{TeV}$ ) and consider the resonance of $A$ which enhances the annihilation of dark matter. The relavent interactions for the $A$-funnel annihilation processes are given by

$$
\begin{aligned}
& \frac{i e}{\sqrt{2} s_{W}} \bar{\chi}_{i}\left[\left(Z_{H}^{11} Z_{-}^{2 i} Z_{+}^{1 j}+Z_{H}^{21} Z_{-}^{1 i} Z_{+}^{2 j}\right) P_{L}\right. \\
& \left.-\left(Z_{H}^{11} Z_{-}^{2 j \star} Z_{+}^{1 i \star}+Z_{H}^{21} Z_{-}^{1 j \star} Z_{+}^{2 i \star}\right) P_{R}\right] \chi_{j} A \\
& -\frac{i e}{2 s_{W} c_{W}} \bar{\chi}_{i}^{0}\left[\left(Z_{H}^{11} Z_{N}^{3 j}-Z_{H}^{21} Z_{N}^{4 j}\right)\left(Z_{N}^{1 i} s_{W}-Z_{N}^{2 i} c_{W}\right) P_{L}\right. \\
& \left.-\left(Z_{H}^{11} Z_{N}^{3 i \star}-Z_{H}^{21} Z_{N}^{4 i \star}\right)\left(Z_{N}^{1 j \star} s_{W}-Z_{N}^{2 j \star} c_{W}\right) P_{R}\right] \chi_{j}^{0} A
\end{aligned}
$$

Where $Z_{H}^{11}=Z_{H}^{22}=\sin \beta, Z_{H}^{12}=-Z_{H}^{21}=-\cos \beta$. At $\mathrm{TeV}$ mass region bino LSP relic density usually highly over abundant, when its mass is about the two times of $A$ Higgs Mass, annihilation cross section soar up due to the $s$-channel resonance. Along with $\tan \beta$, coupling between two LSP and $A$ higgs becomes bigger, annihilation of DM becomes more efficient. For higgsino LSP, two lightest neutralino or two lightest chargino annihilation through $s$-channel $A$ higgs, as a result heavier higgsino LSP also meet correct relic density.

3. Coannihilation cases When a stop or squark has a mass approaching the LSP, it coannihilates with the LSP and helps to achieve the correct relic density. For stop (squark) coannihilation case, $\left|A_{t}\right|<5 \mathrm{TeV}\left(\left|A_{t}\right|,\left|A_{b}\right|<5 \mathrm{TeV}\right)$, the squark (stop) mass is required to within $120 \%$ of the LSP mass, and fix $A$ at $10 \mathrm{TeV}$, The relevant interactions for squark coannihilation are given by 


\section{decoupled}

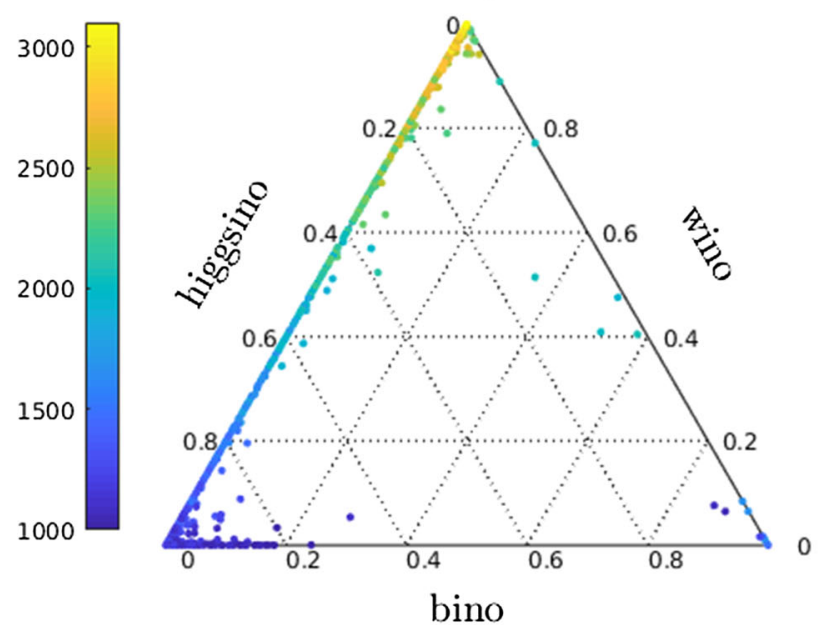

stop coannihilation

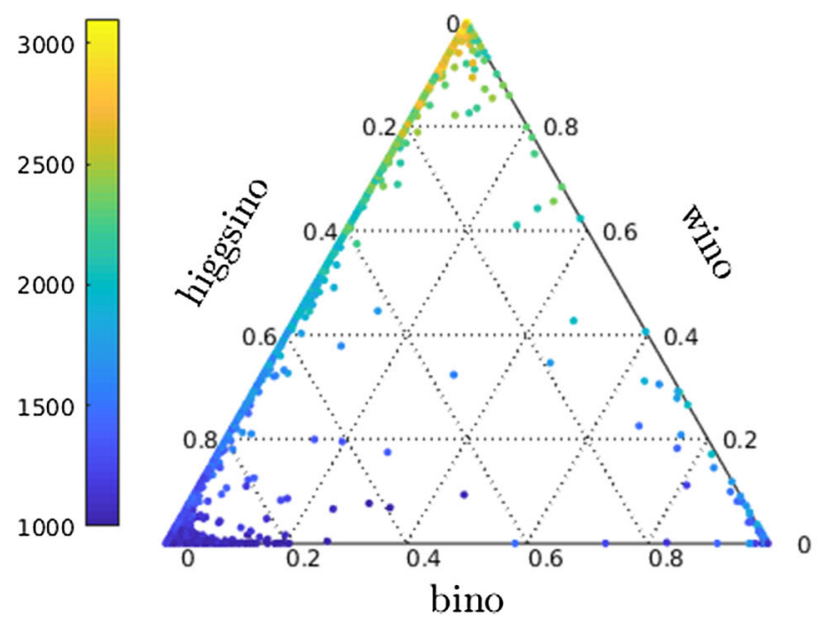

A-mediated

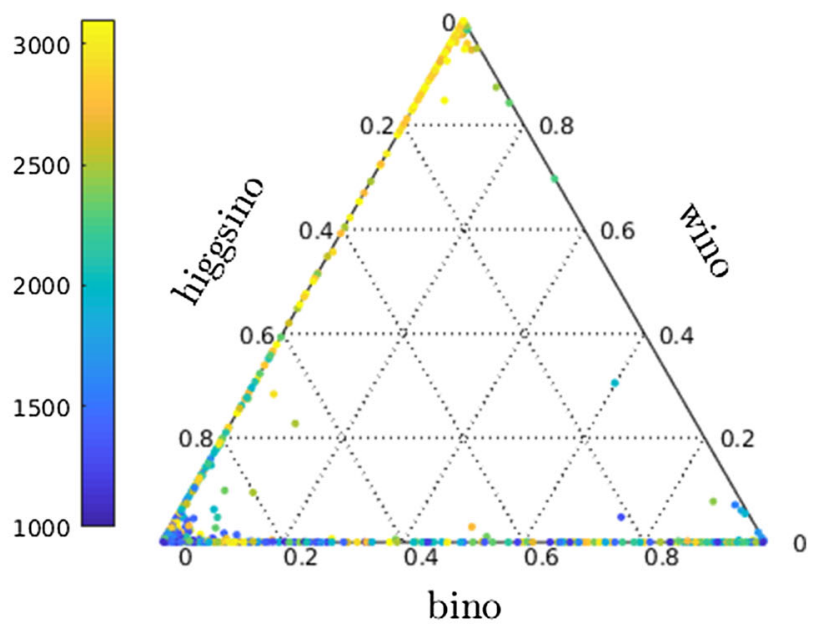

squark coannihilation

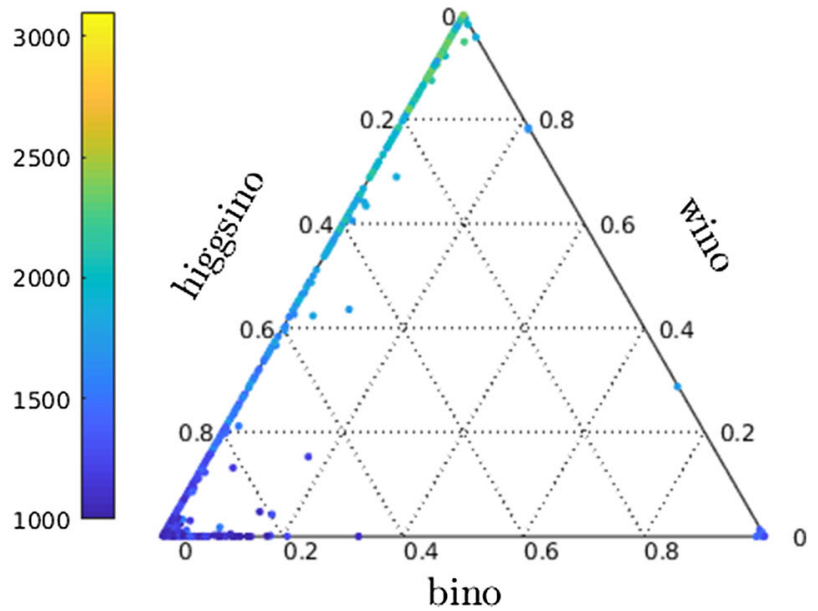

Fig. 1 Scatter plots of the TeV neutralino dark matter showing the components under the requirement of thermal relic density. The colors denote the dark matter mass from 1 to $3.1 \mathrm{TeV}$

$$
\begin{aligned}
& U_{i}^{-} \bar{\chi}_{j}^{0}\left[\left(\frac{-e}{\sqrt{2} s_{W} c_{W}} Z_{U}^{I i \star}\left(\frac{1}{3} Z_{N}^{1 j} s_{W}+Z_{N}^{2 j} c_{W}\right)\right.\right. \\
& \left.-Y_{u}^{I} Z_{U}^{(I+3) 1 \star} Z_{N}^{4 j}\right) P_{L}+\left(\frac{2 e \sqrt{2}}{3 c_{W}} Z_{U}^{(I+3) i \star} Z_{N}^{1 j \star}\right. \\
& \left.\left.-Y_{u}^{I} Z_{U}^{I i \star} Z_{N}^{4 j \star}\right) P_{R}\right] u^{I}+\text { H.c. } \\
& +D_{i}^{+} \bar{\chi}_{j}^{0}\left[\left(\frac{-e}{\sqrt{2} s_{W} c_{W}} Z_{D}^{I i}\left(\frac{1}{3} Z_{N}^{1 j} s_{W}-Z_{N}^{2 j} c_{W}\right)\right.\right. \\
& \left.+Y_{d}^{I} Z_{D}^{(I+3) i} Z_{N}^{3 j}\right) P_{L} \\
& \left.+\left(\frac{-e \sqrt{2}}{3 c_{W}} Z_{D}^{(I+3) i} Z_{N}^{1 j \star}+Y_{d}^{I} Z_{D}^{I i} Z_{N}^{3 j \star}\right) P_{R}\right] d^{I}+\text { H.c. }
\end{aligned}
$$

where $I$ runs from 1 to 3 is three matter generation. $\mu^{I}$ and $d^{I}$ are up and down quarks. $Y_{\mu}^{I}$ and $Y_{d}^{I}$ are Yukawa couplings. Relations between $U^{I}\left(D^{I}\right)$ (up(down) squarks), definition of $U_{i}^{-}\left(D_{i}^{+}\right)$and $Z_{U}\left(Z_{D}\right)$ is somewhat lenghy, it can be found in Ref. [20]. Sign of $Z_{N}^{2 j}$ in third line of Eq. 4 is minus, thus second interaction term's contribution to annihilation cross section is opposite to the first interaction term, in other words, wino LSP mass which satisfy correct relic density becomes smaller.

In Fig. 1 we show the components of the TeV neutralino dark matter under the requirement of correct thermal relic density. We see that in all four cases the $\mathrm{TeV}$ neutralino dark matter is dominated by higgsino or wino in order to satisfy the relic density. Actually, a pure higgsino (wino) dark matter around 1.1 (2.8) TeV (with Sommerfeld enhancement) has been found to give the required thermal relic density in 
the literature $[8,22,23]$. The number of bino-like samples is quite small in the decoupled scenario, but increases in the A-mediation and stop(squark) coannihilation cases, as expected(as in 2 and 3 or 1).

\section{Constraints from dark matter detections}

The on-going direct and indirect detection experiments have constrained the dark matter interactions with the standard model (SM) particles. In Figs. 2 and 3 the upper panels show the spin-independent neutralino-nucleon scattering cross sections where the current upper limits from PandaX and the future sensitivity of LZ7.2T are plotted. We see that the current PandaX data has excluded the region where higgsino and wino are mixed.

The lower panels of Figs. 2 and 3 show the neutralino dark matter annihilation cross sections where the upper limits
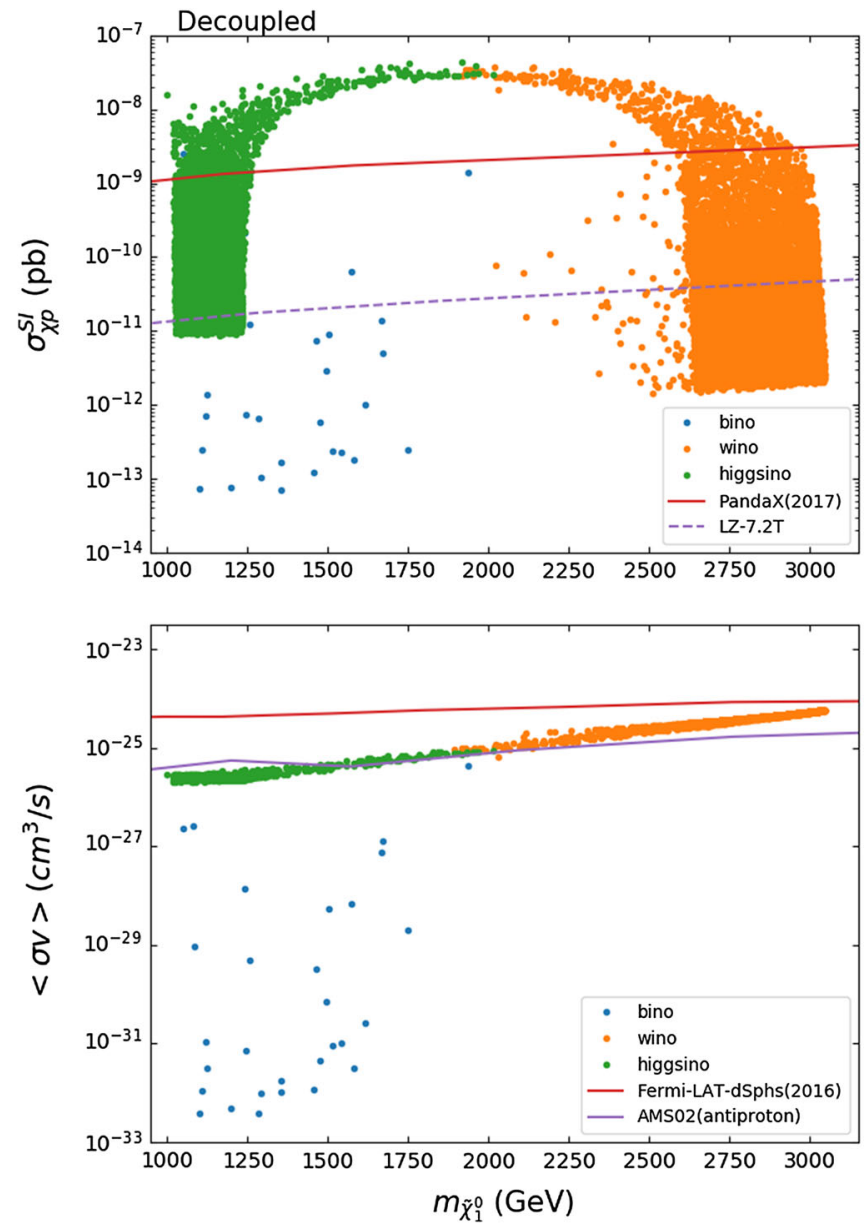

Fig. 2 Scatter plots of parameter space satisfying the relic density at $2 \sigma$ level. The left panels are for the decoupled case while the right panels are for the $A$-mediated case. The upper parts show the spin-independent neutralino LSP-nucleon scattering cross sections where the curves are the 90\% CL upper limits from PandaX (2017) [1] and the future sensi- from the AMS-02 anti-proton data and the Fermi-LAT $\gamma$-ray data [24] from the observation of dwarf spheroidal galaxies are plotted. We see that the upper limits from these indirect detection data are weaker than the direct detection limits (the parameter space above the AMS-02 anti-proton limits has already been excluded by the PandaX data).

In our calculation of the electron/positron and antiproton flux from the dark matter halo, we changed the energy spectrum of dark matter source term in GALPROP [26]. This source term is

$q_{\bar{p}, e^{ \pm}}^{D M}\left(\mathbf{x}, E_{k i n}\right)=\frac{1}{2}\left(\frac{\rho(\mathbf{x})}{m_{D M}}\right)^{2} \sum_{f}\langle\sigma v\rangle_{f} \frac{d N_{\bar{p}, e^{ \pm}}^{f}}{d E_{k i n}}$

where $\bar{p}, e^{ \pm}$are antiproton and electron/positron, $\rho(\mathbf{x})$ is the dark matter density distribution, $m_{D M}$ is the dark matter mass, $\langle\sigma v\rangle_{f}$ is thermally averaged cross section for dark matter annihilation into the $\mathrm{SM}$ final state $f(\mathrm{DM}+\mathrm{DM} \rightarrow$
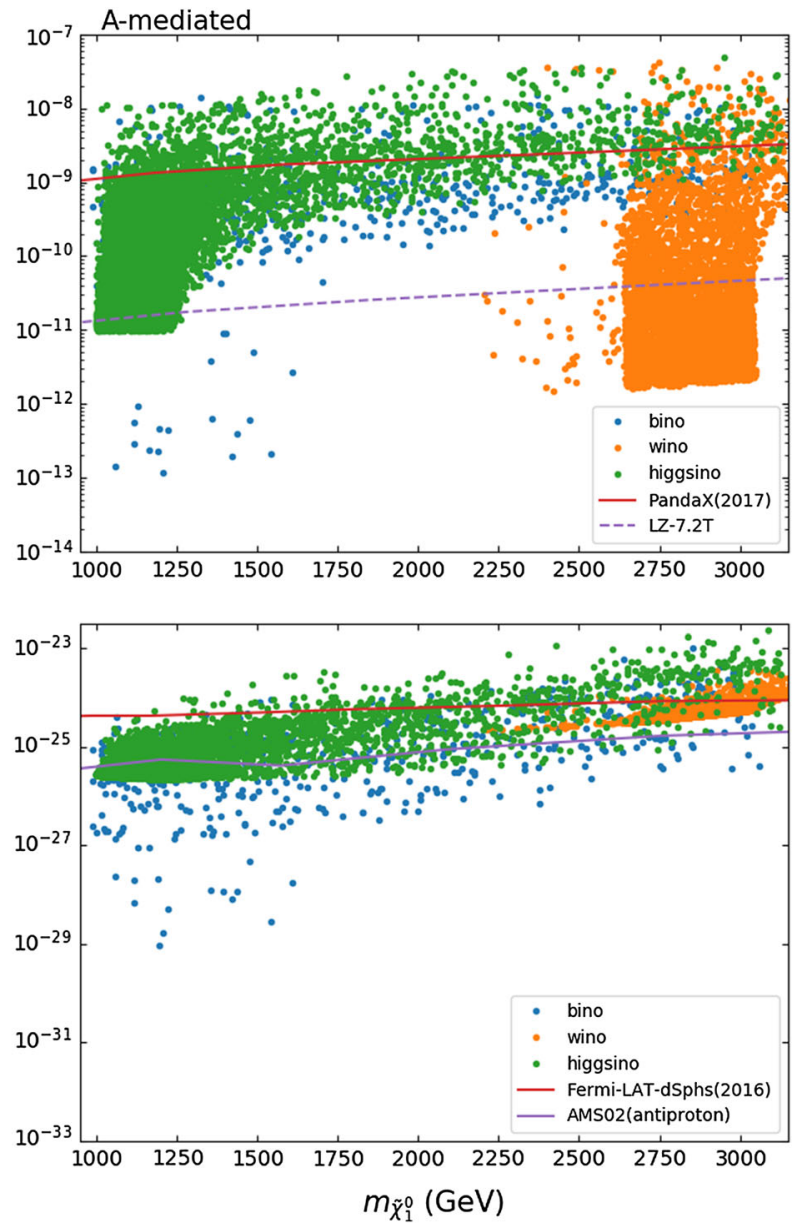

tivity from LZ7.2T. The lower parts show the dark matter annihilation cross sections $\langle\sigma v\rangle$ where the curves are the 95\% CL upper limits from the AMS-02 anti-proton data [25] and the Fermi-LAT $\gamma$-ray data (dwarf spheroidal galaxies) [24]. The bino, wino and higgsino samples represent bino-like, wino-like and higgsino-like LSP, respectively 

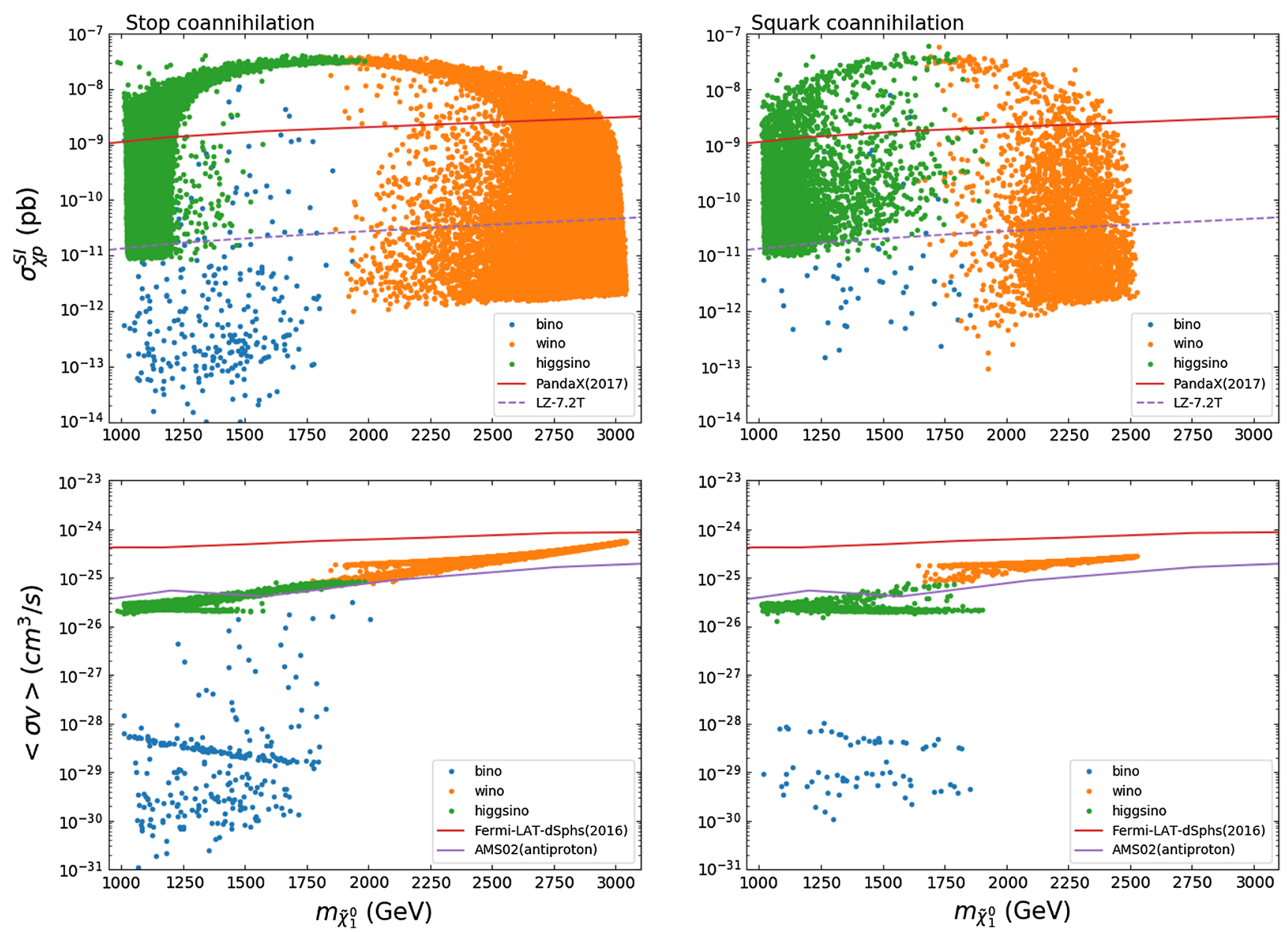

Fig. 3 Same as Fig. 2, but for squark and stop coannihilation cases
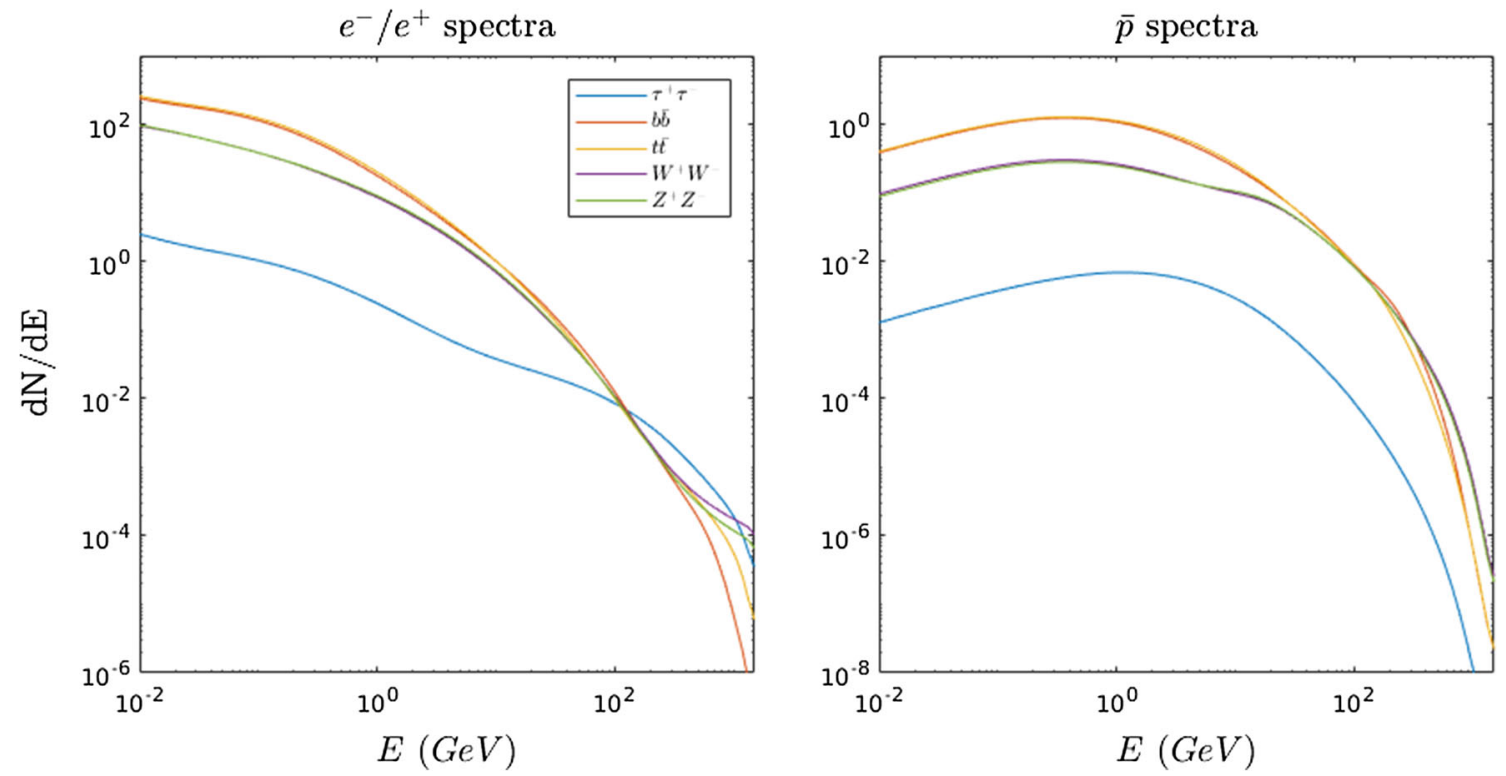

Fig. 4 The positron/electron and antiproton spectra produced by the annihilation of two dark matter particles with mass $1.5 \mathrm{TeV}$ 
Table 1 Four benchmark points, one point for each case (decoupled case, $A$-mediated case, squark and stop coannihilation cases) with largest $\langle\sigma v\rangle$

\begin{tabular}{|c|c|c|c|c|c|c|c|c|c|}
\hline \multirow[t]{2}{*}{$m_{\mathrm{DM}}(\mathrm{GeV})$} & \multirow[t]{2}{*}{$\langle\sigma v\rangle\left(\mathrm{cm}^{3} / \mathrm{s}\right)$} & \multicolumn{5}{|c|}{ DM annihilation final states ratio $(f \bar{f})$} & \multicolumn{3}{|c|}{ DM components } \\
\hline & & $\overline{W^{+} W^{-}}$ & $Z^{+} Z^{-}$ & $\tau^{+} \tau^{-}$ & $b \bar{b}$ & $t \bar{t}$ & Bino & Wino & $\overline{\text { Higgsino }}$ \\
\hline 1111 & $2.4 \times 10^{-26}$ & 0.57 & 0.43 & 0 & 0 & 0 & 0 & 0 & 1.0 \\
\hline 1584 & $4.3 \times 10^{-26}$ & 0.71 & 0.14 & 0 & 0 & 0.15 & 0 & 0.22 & 0.78 \\
\hline 2077 & $9.1 \times 10^{-26}$ & 0.18 & 0.01 & 0.15 & 0.80 & 0.01 & 0 & 0.05 & 0.95 \\
\hline 3012 & $1.3 \times 10^{-25}$ & 0 & 0 & 0.17 & 0.82 & 0 & 0.17 & 0 & 0.83 \\
\hline
\end{tabular}

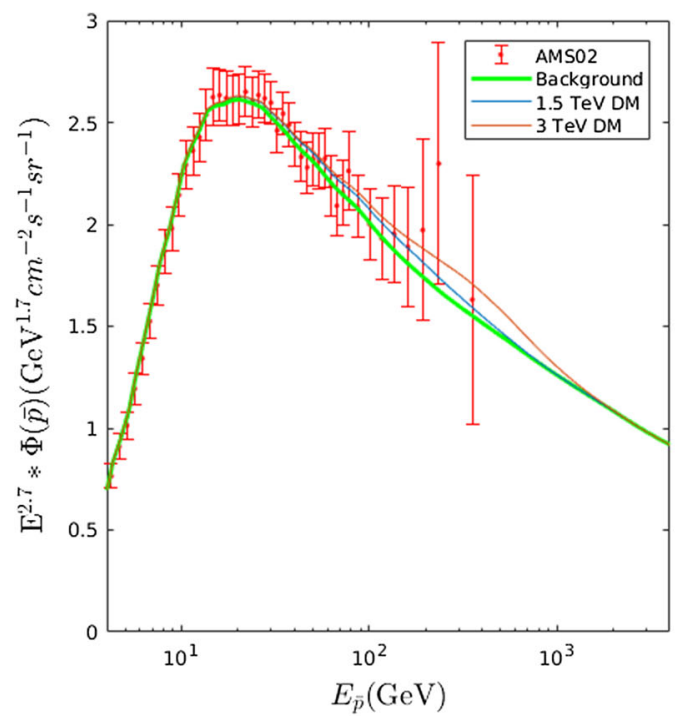

Fig. 5 The antiproton flux (left panel) and electron plus positron flux (right panel). For the antiproton flux, the AMS-02 data [30] and its fitted curve as well as the results with the $1.5 \mathrm{TeV}$ neutralino dark matter annihilation contribution are shown. For the electron plus positron flux,

$f \bar{f}), d N_{\bar{p}, e^{ \pm}}^{f} / d E_{k i n}$ are the antiproton and eletron/positron energy spectrum per annihilation, and the factor $1 / 2$ is for the Majorana dark matter fermion. For $\rho(\mathbf{x})$ we use NFW dark matter density profile [27]

$\rho_{N F W}(r)=\rho_{0} \frac{r_{0}}{r}\left(\frac{r_{0}}{r_{0}+r}\right)^{2}$

with the halo radius $20 \mathrm{kpc}$, the local dark matter density $\rho_{0}$ $=0.43 \mathrm{GeV} / \mathrm{cm}^{3}[28]$ at the solar position $r_{0}=8 \mathrm{kpc}$.

From the source term we know that $m_{D M}$ and $\langle\sigma v\rangle_{F}$ are crucial for the intensity of the flux and, therefore, we here choose one benchmark point for each case. The energy spectrum $d N_{\bar{p}, e^{ \pm}}^{f} / d E_{k i n}$ data from [29] are used with the interpolation method for the source term in GALPROP. In the 1-3 $\mathrm{TeV}$ dark matter mass range, the spectra of $e^{ \pm}, \bar{p}$ are similar and thus we only show the spectra for $m_{D M}=1.5 \mathrm{TeV}$ in Fig. 4. Also, we display in Table 1 four benchmark points, one point for each case with largest $\langle\sigma v\rangle$.

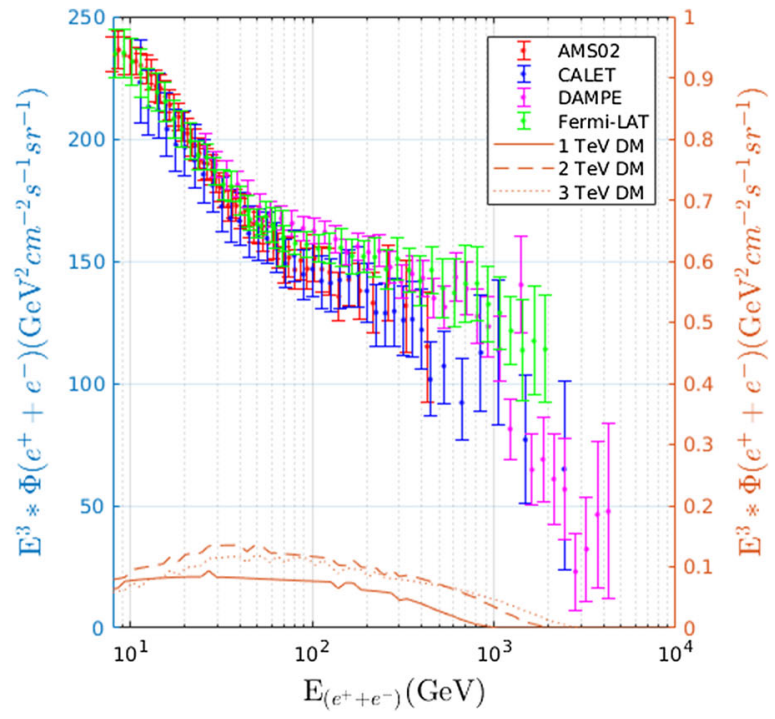

the AMS-02 [31], the CALET [32], the DMAPE [2] and the Fermi-LAT [33] data (read the left Y-axis) as well as the 1-3 TeV neutralino dark matter annihilation contribution (read the right Y-axis) are shown. The errors of the data are $1 \sigma$ statistical and systematic

Figure 5 is the antiproton and electron plus positron flux calculated by GALPROP, compared with the experimental data. Here we see that the contributions of the 1-3 TeV neutralino dark matter annihilation are too small to cause visible excess. This means that the plausible electron/positron cosmic-ray excess at $\mathrm{TeV}$ energy reported by DAMPE [2] is not likely from the $\mathrm{TeV}$ neutralino dark matter annihilation. If this excess is verified, it may point to some TeV leptophilic dark matter [34]. However, the small contribution to the antiproton flux from the $\mathrm{TeV}$ neutralino dark matter annihilation is still favored because so far no excess has been observed for the antiproton flux.

In summary, the $1-3 \mathrm{TeV}$ neutralino dark matter with correct thermal relic density has been stringently constrained by the direct detection data. The constraints of indirect detections from cosmic-ray flux are much weaker than direct detection limits. The survived parameter space can be mostly covered by the future direct detection experiment LZ7.2T. At the colliders, the $\mathrm{TeV}$ neutralino dark matter is hard to probe 
at the LHC [35], but can be effectively probed at a $100 \mathrm{TeV}$ hadron collider. For example, for a luminosity of $3000 \mathrm{fb}^{-1}$, a $100 \mathrm{TeV}$ hadron collider can give a good probe for a $\mathrm{TeV}$ higgsino in the decoupled case [36] and $1-3 \mathrm{TeV}$ for stop coannihilation case [37].

\section{Conclusion}

In this work we examined the thermal neutralino dark matter in a mass range of $1-3 \mathrm{TeV}$. We considered various scenarios and confronted them with the latest direct and indirect detections from PandaX and AMS-02/DAMPE. We observed that the parameter space is stringently constrained by the direct detection limits. In the allowed parameter space, the $1-3 \mathrm{TeV}$ neutralino dark matter annihilation contribution to the antiproton flux is found to agree with the AMS-02 data while its contribution to eletron/positron flux is too small to cause any visible excess.

Acknowledgements This work was supported by the National Natural Science Foundation of China (NNSFC) under Grant nos. 11705093, 11305049, 11675242 and 11375001, by Peng-Huan-Wu Theoretical Physics Innovation Center (11747601), by the CAS Center for Excellence in Particle Physics (CCEPP), by the CAS Key Research Program of Frontier Sciences and by a Key R\&D Program of Ministry of Science and Technology of China under number 2017YFA0402200-04.

Data Availability Statement This manuscript has no associated data or the data will not be deposited. [Authors' comment: Data is lost].

Open Access This article is distributed under the terms of the Creative Commons Attribution 4.0 International License (http://creativecomm ons.org/licenses/by/4.0/), which permits unrestricted use, distribution, and reproduction in any medium, provided you give appropriate credit to the original author(s) and the source, provide a link to the Creative Commons license, and indicate if changes were made. Funded by SCOAP . $^{3}$

\section{References}

1. X. Cui et al. (PandaX-II), Phys. Rev. Lett. 119, 181302 (2017). arXiv: 1708.06917 [astro-ph.CO]

2. G. Ambrosi et al. (DAMPE), Nature 552, 63 (2017). arXiv:1711.10981 [astro-ph.HE]

3. G.H. Duan, W. Wang, L. Wu, J.M. Yang, J. Zhao, Phys. Lett. B 778, 296 (2018a). arXiv:1711.03893 [hep-ph]

4. J. Ren, L. Wu, J.M. Yang, J. Zhao ( 2017). arXiv:1708.06615 [hep$\mathrm{ph}]$

5. M. Beneke, A. Bharucha, F. Dighera, C. Hellmann, A. Hryczuk, S. Recksiegel, P. Ruiz-Femenia, JHEP 03, 119 (2016). arXiv:1601.04718 [hep-ph]

6. M. Beneke, A. Bharucha, A. Hryczuk, S. Recksiegel, P. RuizFemenia, JHEP 01, 002 (2017). arXiv:1611.00804 [hep-ph]

7. M. Abdughani, L. Wu, J.M. Yang, Eur. Phys. J. C 78, 4 (2018). arXiv:1705.09164 [hep-ph]

8. J. Bramante, N. Desai, P. Fox, A. Martin, B. Ostdiek, T. Plehn, Phys. Rev. D 93, 063525 (2016). arXiv:1510.03460 [hep-ph]
9. G.H. Duan, K.-I. Hikasa, J. Ren, L. Wu, J.M. Yang. Probing binowino coannihilation dark matter below the neutrino floor at the LHC. Phys. Rev. D98(1), 015010 (2018). arXiv:1804.05238 [hep$\mathrm{ph}]$

10. C. Han, J. Ren, L. Wu, J.M. Yang, M. Zhang, Eur. Phys. J. C 77, 93 (2017a). arXiv:1609.02361 [hep-ph]

11. A. Kobakhidze, M. Talia, L. Wu, Phys. Rev. D 95, 055023 (2017). arXiv:1608.03641 [hep-ph]

12. N. Liu, L. Wu, Eur. Phys. J. C 77, 868 (2017). arXiv:1705.02534 [hep-ph]

13. C. Han, K.-I. Hikasa, L. Wu, J.M. Yang, Y. Zhang, Phys. Lett. B 769, 470 (2017b). arXiv:1612.02296 [hep-ph]

14. G. Grilli di Cortona, JHEP 05, 035 (2015). arXiv:1412.5952 [hep$\mathrm{ph}]$

15. K. Kowalska, E.M. Sessolo. The discreet charm of higgsino dark matter - a pocket review. Adv. High Energy Phys. 2018, 6828560 (2018). arXiv:1802.04097 [hep-ph]

16. A. Mitridate, M. Redi, J. Smirnov, A. Strumia, JCAP 1705, 006 (2017). arXiv:1702.01141 [hep-ph]

17. G. Belanger, F. Boudjema, A. Pukhov, A. Semenov, Comput. Phys. Commun. 185, 960 (2014). arXiv:1305.0237 [hep-ph]

18. P.A.R. Ade et al. (Planck), Astron. Astrophys. 571, A16 (2014). arXiv:1303.5076 [astro-ph.CO]

19. J.D. Wells, in 11th International Conference on Supersymmetry and the Unification of Fundamental Interactions (SUSY 2003) Tucson, Arizona, June 5-10, 2003 (2003). arXiv:hep-ph/0306127 [hep$\mathrm{ph}]$

20. J. Rosiek. Complete set of Feynman rules for the MSSM: Erratum (1995). arXiv:hep-ph/9511250 [hep-ph]

21. S.P. Martin, 1 (1997) [Adv. Ser. Direct. High Energy Phys. 18, 1 (1998)]. arXiv:hep-ph/9709356 [hep-ph]

22. T. Cohen, M. Lisanti, A. Pierce, T.R. Slatyer, JCAP 1310, 061 (2013). arXiv:1307.4082 [hep-ph]

23. J. Fan, M. Reece, JHEP 10, 124 (2013). arXiv:1307.4400 [hep-ph]

24. A. Albert et al. (DES, Fermi-LAT), Astrophys. J. 834, 110 (2017). arXiv:1611.03184 [astro-ph.HE]

25. A. Cuoco, J. Heisig, M. Korsmeier, M. Krämer. Constraining heavy dark matter with cosmic-ray antiprotons. JCAP 1804(4), 4 (2018). arXiv:1711.05274 [hep-ph]

26. A.W. Strong, I.V. Moskalenko, O. Reimer, Astrophys. J. 537, 763 ( 2000) [Erratum: Astrophys. J. 541, 1109 (2000)]. arXiv:astro-ph/9811296 [astro-ph]

27. J.F. Navarro, C.S. Frenk, S.D.M. White, Astrophys. J. 462, 563 (1996). arXiv:astro-ph/9508025 [astro-ph]

28. P. Salucci, F. Nesti, G. Gentile, C.F. Martins, Astron. Astrophys. 523, A83 (2010). arXiv:1003.3101 [astro-ph.GA]

29. M. Cirelli, G. Corcella, A. Hektor, G. Hutsi, M. Kadastik, P. Panci, M. Raidal, F. Sala, A. Strumia, JCAP 1103, 051 (2011) [Erratum: JCAP 1210, E01 (2012)]. arXiv:1012.4515 [hep-ph]

30. M. Aguilar et al. (AMS), Phys. Rev. Lett. 117, 091103 (2016)

31. M. Aguilar et al. (AMS), Phys. Rev. Lett. 113, 121102 (2014)

32. O. Adriani et al., (CALET). Phys. Rev. Lett. 119, 181101 (2017). arXiv:1712.01711 [astro-ph.HE]

33. S. Abdollahi et al., (Fermi-LAT). Phys. Rev. D 95, 082007 (2017). arXiv:1704.07195 [astro-ph.HE]

34. G.H. Duan, L. Feng, F. Wang, L. Wu, J.M. Yang, R. Zheng, JHEP 02, 107 (2018c). arXiv:1711.11012 [hep-ph]

35. C. Han, A. Kobakhidze, N. Liu, A. Saavedra, L. Wu, J. M. Yang, JHEP 02, 049 (2014), arXiv:1310.4274 [hep-ph]

36. M. Low, L.-T. Wang, JHEP 08, 161 (2014). arXiv:1404.0682 [hep$\mathrm{ph}]$

37. T. Cohen, R.T. D' Agnolo, M. Hance, H.K. Lou, J.G. Wacker, JHEP 11, 021 (2014). arXiv:1406.4512 [hep-ph] 